\title{
Determinação do nível de dano econômico de tripes em cebola.
}

\author{
Paulo A. S. Gonçalves \\ EPAGRI S.A., C. Postal 121, 88400-000 Ituporanga - SC.
}

RESUMO

Com o objetivo de se determinar o nível de dano econômico de Thrips tabaci Lind. em cebola foram realizados dois experimentos na EPAGRI S.A., Ituporanga, SC, entre agosto e dezembro de 1993 e 1995. Em 1993 aplicações de lambdacialotrina 5 g. i.a/ha foram realizadas quando o número médio de ninfas de tripes por planta foi igual ou superior a 5, 7, 10 e 15, durante todo o ciclo da cultura; quando o número médio de ninfas foi igual 5,7 até a formação do bulbo e 10,15, 20 após; 10 até a formação do bulbo e 20, 30 após. Em 1995 aplicações de lambacialotrina $30 \mathrm{~g}$ i.a/ha foram realizadas quando o nível médio de tripes/planta foi: $1,3,5,7,10,15,20,25$, 30, 40, 50, 60, e testemunha. Em 1993 foi possível reduzir o número de pulverizações de oito (controle adotado sempre que o nível populacional de tripes atingisse 5 ninfas/planta) para quatro (tratamento com 10 ninfas antes e 30 após a formação do bulbo) sem prejudicar a produtividade. Em 1995 o número de pulverizações variou de zero para o nível médio igual ou superior a 20 ninfas/ planta até sete para o nível de 1 ninfa/planta e a produtividade foi igual entre tratamentos. Sendo assim, as plantas de cebola toleraram o dano de tripes sem reduzir a produtividade. Isto ocorreu devido ao manejo do solo (em 1995 além de adubação química foi adicionado adubação verde com mucuna, Stilozobium sp., e orgânica com esterco de aves) e a distribuição de precipitação pluviométrica mais regular. Portanto, o nível de dano econômico de tripes em cebola está relacionado com práticas adequadas de manejo de solo e distribuição de precipitação pluviométrica.

Palavras-chave: Allium cepa, Insecta, (Thrips tabaci) tripes da cebola, controle cultural, dano.

\begin{abstract}
Determination of economic injury level of onion thrips.

The objectives of this work to determine the economic injury level of Thrips tabaci Lind. on onion. Two experiments were carried out at EPAGRI S.A., Ituporanga, Santa Catarina State, Brazil, between August and December, 1993 and 1995. In 1993, sprays with lambdacyhalothrin, $5 \mathrm{~g}$ a.i/ha, were used throught the cycle whenever the number of nymphs of onion thrips reached the following levels per plant: $5,7,10$ and 15 , during the whole cycle; 5 , and 7 by bulb development and 10,15, 20 after; 10 by bulb development and 20 , 30 after; and untreated control. In 1995, spray with lambdacyhalothrin, $30 \mathrm{~g} \mathrm{a}$. $/$ ha, was used when the levels of nymphs per plant were observed during the whole cycle: $1,3,5,7,10,15,20,25,30,40$, 50,60 and untreated control. In 1993, the number of sprays was reduced from eight (using chemical control when the number of 5 nymphs per plant was reached) to four (treatments with 10 nymphs before and 30 after the bulb development). In 1995, the untreated control gave a similar yield to chemical control treatments. Hence the plants tolerated the onion thrips damage. This difference was due to soil management (in 1995, in addition to the chemical fertilizer, green manuring with Stilozobium sp was also used) and to regular distribution of precipitation. Therefore the economic injury level of onion thrips is related to rational soil management and distribution of precipitation.
\end{abstract}

Keywords: Allium cepa, Thrips tabaci, insecta, onion thrips, cultural control, damage.

(Aceito para publicação em 01 de setembro de 1998)

\begin{abstract}
$\mathrm{A}$ principal praga da cebola em San ta Catarina é o tripes, Thrips tabaci Lind. (EMPASC/ACARESC, 1991). Os agricultores da região do Alto Vale do Itajaí, SC, têm utilizado para o controle desta praga inseticidas fosforados e piretróides, principalmente parathion metílico e deltamethrina (Gonçalves \& Guimarães, 1995). Recentemente, temse incrementado a utilização de cipermetrina, lambdacialotrina, metamidofós e clorpirifós-etil pelos produtores de cebola catarinenses. $\mathrm{O}$ número de pulverizações/safra pode atingir até doze em alguns casos. Este procedimento pode gerar a médio prazo problemas de saúde ao aplicador de agrotóxicos, a contaminação do meio ambiente e do produto final, além do ônus econômi-
\end{abstract}

co. Com o advento do MERCOSUL, o agricultor deve racionalizar os custos, a fim de obter produtividade e qualidade na produção de bulbos para competir com o mercado estrangeiro, sobretudo $o$ argentino.

Os meses de outubro e novembro foram considerados como sendo os de maior ocorrência de tripes na região do Alto Vale do Itajaí, SC, com infestações severas a partir da segunda quinzena de outubro (Lorini et al., 1986; Gonçalves \& Guimarães, 1995). A realização de pulverizações periódicas nesta fase não produzem necessariamente incrementos na produtividade (Gonçalves, 1996). Lorini \& Ferreto (1991) não observaram diferença no rendimento de cebola ao utilizarem aplicações de deltametrina
7,5 g i.a/ha em intervalos de 3, 7, 14 e 21 dias. Os trabalhos que relacionam número de tripes por planta e produtividade são polêmicos, pois fatores como fenologia, épocas de plantio e de infestações, variedade e clima podem influenciar na interação inseto-planta (Domiciano et al., 1993). Diante disso, é necessário considerar as características de cada região produtora (clima, época de plantio, cultivar utilizada) para se determinar a relação entre densidade populacional de tripes e rendimento da cultura. Hoffman et al. (1995) observaram uma redução do uso de inseticidas sem afetar a qualidade e a produtividade de cebola ao adotarem o nível de dano econômico para o manejo de tripes nesta cultura. A adoção desta estratégia de 
manejo de tripes contribuiria para a redução de custos de produção, problemas ambientais e aqueles relacionados à saúde do agricultor catarinense.

Este trabalho teve como objetivo determinar o nível de dano econômico de tripes em cebola, visando fornecer informações que possibilitem o manejo mais racional da cultura.

\section{MATERIAL E MÉTODOS}

O trabalho foi conduzido na Estação Experimental de Ituporanga, SC, EPAGRI S.A., a $475 \mathrm{~m}$ de altitude, em cambissolo húmico distrófico álico. Os dois experimentos foram conduzidos em 1993 (20/ 08 a 7/12) e 1995 ( 22/08 a 18/12).

A cultivar de cebola EMPASC 351 Sel. Crioula, utilizada nos dois ensaios, foi manejada de acordo com as recomendações propostas por EMPASC/ ACARESC (1991). A adubação no ensaio de 1993 foi de $1000 \mathrm{~kg} / \mathrm{ha}$ da formulação N-P-K, 5-20-10. Em 1995, além de $800 \mathrm{~kg} /$ ha desta formulação, utilizou-se adubação verde prévia com mucuna (Stilozobium sp.) e adubação orgânica com esterco de aves (15 t/ha) incorporada 21 dias antes do transplante.

O delineamento foi blocos ao acaso com treze tratamentos e quatro repetições. O tamanho da parcela foi de $2,8 \mathrm{~m}$ x 3,0m, espaçamento de $40 \mathrm{~cm} \mathrm{x} \mathrm{7,5} \mathrm{cm,}$ com 280 plantas/parcela, área útil de 6 $\mathrm{m}^{2}$, em ambos os experimentos.

Em 1993, a aplicação de lambdacialotrina (5 g i.a/ha) foi realizada quando o número médio de ninfas de tripes foi igual ou superior a: (1) 5; (2) 7 ; (3) 10 e (4) 15 ninfas, durante todo o ciclo da cultura; (5), (6) e (7) 5 ninfas até a formação do bulbo e 10, 15, 20 após; (8), (9) e (10) 7 ninfas até a formação do bulbo e 10, 15, 20 após; (11) e (12) 10 ninfas até a formação do bulbo 20 e 30 após e (13) testemunha sem aplicação. O período compreendido entre o transplante até a primeira quinzena de novembro foi considerado como estádio de formação do bulbo.

Em 1995, a aplicação de lambdacialotrina (30 $\mathrm{g}$ i.a/ha) foi realizada quando a densidade populacional atingiu os seguintes níveis de ninfas de
Tabela 1. Número de pulverizações de inseticida no controle de tripes, Thrips tabaci, em cebola e produtividade da cv. EMPASC 351 Sel. Crioula. Ituporanga, EPAGRI S/A, 1993.

\begin{tabular}{lcc}
\hline \multicolumn{1}{c}{ Tratamentos } & Pulverizações ( no) & Produtividade (t/ha)* \\
\hline 5 ninfas & 8 & $17,4 \mathrm{a}$ \\
7 ninfas & 6 & $14,2 \mathrm{a}$ \\
10 ninfas & 6 & $13,3 \mathrm{a}$ \\
15 ninfas & 6 & $14,7 \mathrm{a}$ \\
5 ninfas/10 ninfas** & 7 & $17,4 \mathrm{a}$ \\
5 ninfas/15 ninfas** & 7 & $17,2 \mathrm{a}$ \\
5 ninfas/20 ninfas** & 7 & $15,6 \mathrm{a}$ \\
7 ninfas/10 ninfas** & 6 & $13,1 \mathrm{a}$ \\
7 ninfas/15 ninfas** & 6 & $13,2 \mathrm{a}$ \\
7 ninfas/20 ninfas** & 5 & $17,3 \mathrm{a}$ \\
10 ninfas/20 ninfas** & 5 & $14,8 \mathrm{a}$ \\
10 ninfas/30 ninfas** & 4 & $12,2 \mathrm{a}$ \\
Testemunha & 0 & $5,1 \mathrm{~b}$ \\
\hline
\end{tabular}

* Médias seguidas da mesma letra não diferem entre si ao nível de $5 \%$ de probabilidade pelo teste de Duncan.

** Os tratamentos com dois níveis populacionais indicam valores de densidade populacional permitida antes e após a formação do bulbo.

tripes/planta durante o ciclo da cultura: (1) 1 ; (2) 3 ; (3) 5 ; (4) 7 ; (5) 10 ; (6) 15 ; (7) 20 ; (8) 25; (9) 30; (10) 40; (11) $50 \mathrm{e}$ (12) 60 ninfas por planta, e (13) testemunha sem aplicação. $\mathrm{O}$ equipamento utilizado na aplicação do inseticida foi um pulverizador costal manual, com bico leque 80.03 (1993) e 110.04 (1995), proporcionando um volume de calda de aproximadamente $600 \mathrm{l} / \mathrm{ha}$.

Para a avaliação do número de tripes coletou-se semanalmente 5 plantas/parcela. Estas foram acondicionadas em sacos de plástico e levadas ao laboratório para as contagens. Para facilitar a avaliação, as plantas foram cortadas à altura do pseudo-caule e as ninfas presentes em todas as folhas foram contadas sob lupa de alça 10x. Em 1993 foram realizadas nove avaliações no período de cinco de outubro (46 dias após transplante - DAP) a 30 de novembro, e em 1995 onze, de vinte e sete de setembro (38 DAP) a quatro de dezembro. O período de avaliações ocorreu principalmente nos meses de outubro e novembro, pois são os meses de maior ocorrência da praga (Lorini et al. 1986; Gonçalves \& Guimarães, 1995). O número médio de ninfas de tripes na testemunha foi correlacionado com valores de precipitação pluviométrica do perío- do de realização do experimento, segundo dados fornecidos pela Estação Agrometeorológica da EPAGRI, Ituporanga, SC. A produtividade foi avaliada em 60 bulbos/parcela, coletados em área de $1,8 \mathrm{~m} \times 1,5 \mathrm{~m}$, previamente delimitada no centro da parcela, onde foram selecionados apenas os bulbos com características comerciais (acima de $4 \mathrm{~cm}$ de diâmetro). Os valores de produtividade foram submetidos à análise de variância e as médias comparadas pelo teste Duncan ao nível de $5 \%$ de probabilidade (Gomes, 1987).

\section{RESULTADOS E DISCUSSÃO}

Em 1993, adotou-se um maior número de pulverizações (oito) para o tratamento com o número médio de até 5 ninfas/planta, enquanto que apenas quatro aplicações foram feitas para o nível de 10 ninfas antes da formação do bulbo e 30 após. Não houve diferença significativa para os valores de produtividade entre os tratamentos com o controle químico (Tabela 1). Portanto, foi possível reduzir o número de pulverizações de oito para quatro mantendo-se níveis semelhantes de produtividade. Em 1995, o número de pulverizações variou de sete para o número médio de 
1 ninfa/planta até zero para níveis acima de 20 ninfas/planta. A produtividade não diferiu significativamente entre tratamentos (Tabela 2). Desta forma, não houve incremento na produtividade devido ao uso de inseticidas. A produtividade da cultura foi influenciada, principalmente, por dois fatores: manejo de solo e precipitação pluviométrica. Comparando-se a produtividade da testemunha obtida em 1993 com aquela de 1995, observou-se, respectivamente $5,1 \mathrm{t} /$ ha e 24,8 t/ha (Tabelas 1 e 2). Este incremento deveu-se à adubação da área experimental, pois em 1995, além da adubação mineral foi utilizado também adubação verde e orgânica. Além disso, a precipitação pluviométrica medida em 1995 (275,1 mm) embora menor que em $1993(309,0 \mathrm{~mm})$ foi regularmente distribuída durante todo o ciclo da cultura, correlacionando-se negativamente com o número médio de ninfas de tripes no tratamento testemunha, favorecendo à redução populacional da praga (por dano físico ao inseto). Entretanto, no experimento de 1993 não houve correlação significativa (Tabela 3). De acordo com Fournier et al. (1995), o déficit hídrico está relacionado com a capacidade de plantas de cebola em tolerar o dano de tripes. Assim, o nível de dano econômico de tripes em cebola está também associado ao estado nutricional da cultura. Domiciano et al., 1993 consideraram outros fatores tais como: fenologia da planta, época de plantio, variedades, clima, níveis de tripes e duração da infestação.

O nível de dano econômico estabelecido por Domiciano et al. (1993) para tripes em cebola, foi $20 \pm 5$ tripes/planta, enquanto que Shelton et al. (1987) adotaram o nível de 3 tripes/folha. Fournier et al. (1995) estimaram os níveis de 2,2 e 0,9 tripes/folha sendo este último adotado para condições de déficit hídrico severo. Adaptando estes níveis para a cv. EMPASC 351 Seleção Crioula, que segundo Yokoyama et al. (1984), pode apresentar no desenvolvimento máximo de 8 a 10 folhas, o controle químico seria adotado entre 7,2 e 30 tripes/planta. Hoffmann et al. (1995) em programa de manejo de tripes na cultura da cebola adotaram os níveis de 1,5 e 3 tripes/folha e não observaram

Tabela 2. Número de pulverizações de inseticida no controle de tripes, Thrips tabaci, em cebola e produtividade da cv. EMPASC 351 Sel. Crioula. Ituporanga, EPAGRI S/A, 1995.

\begin{tabular}{lcc}
\hline \multicolumn{1}{c}{ Tratamentos } & Pulverizações $\left(\mathbf{n}^{\mathbf{0}}\right)$ & Produtividade $(\mathbf{t} / \mathbf{h a})^{*}$ \\
\hline 1 ninfa & 7 & $23,3 \mathrm{a}$ \\
3 ninfas & 7 & $25,0 \mathrm{a}$ \\
5 ninfas & 7 & $23,8 \mathrm{a}$ \\
7 ninfas & 5 & $25,4 \mathrm{a}$ \\
10 ninfas & 5 & $22,0 \mathrm{a}$ \\
15 ninfas & 4 & $27,3 \mathrm{a}$ \\
20 ninfas & 0 & $22,9 \mathrm{a}$ \\
25 ninfas & 0 & $23,6 \mathrm{a}$ \\
30 ninfas & 0 & $21,3 \mathrm{a}$ \\
40 ninfas & 0 & $23,3 \mathrm{a}$ \\
50 ninfas & 0 & $24,7 \mathrm{a}$ \\
60 ninfas & 0 & $23,6 \mathrm{a}$ \\
Testemunha & 0 & $24,8 \mathrm{a}$ \\
\hline
\end{tabular}

* Médias seguidas da mesma letra não diferem entre si ao nível de $5 \%$ de probabilidade pelo teste de Duncan.

Tabela 3. Número médio de ninfas de tripes, Thrips tabaci, por planta na testemunha, precipitação pluviométrica acumulada entre as datas de avaliação, e coeficiente de correlação (r). Ituporanga, EPAGRI S/A,1993/1995.

\begin{tabular}{|c|c|c|c|c|c|c|c|}
\hline \multicolumn{4}{|c|}{1993} & \multicolumn{4}{|c|}{1995} \\
\hline Data & $\begin{array}{l}\text { Precipitação } \\
\text { (mm) }\end{array}$ & Ninfas & $\mathbf{r}$ & Data & $\begin{array}{l}\text { Precipitação } \\
\text { (mm) }\end{array}$ & Ninfas & $\mathbf{r}$ \\
\hline $05 / 10$ & 58,6 & 1,1 & $-0,18 \mathrm{~ns}^{1}$ & $27 / 09$ & 81,0 & 1,8 & $-0,66 \mathrm{~s}^{1}$ \\
\hline $13 / 10$ & 0,0 & 5,9 & & $02 / 10$ & 29,0 & 0,7 & \\
\hline $19 / 10$ & 18,8 & 24,0 & & $09 / 10$ & 19,0 & 4,3 & \\
\hline $26 / 10$ & 69,8 & 32,8 & & $16 / 10$ & 39,0 & 7,0 & \\
\hline $04 / 11$ & 1,4 & 40,1 & & $23 / 10$ & 34,0 & 6,2 & \\
\hline $09 / 11$ & 5,4 & 40,8 & & $30 / 10$ & 24,0 & 18,5 & \\
\hline $17 / 11$ & 47,4 & 15,7 & & $06 / 11$ & 6,3 & 26,5 & \\
\hline $23 / 11$ & 0,0 & 13,4 & & $13 / 11$ & 16,0 & 14,7 & \\
\hline $30 / 11$ & 107,6 & 15,1 & & $20 / 11$ & 7,1 & 20,8 & \\
\hline \multirow[t]{2}{*}{ Total } & 309,0 & & & $27 / 11$ & 16,0 & 19,4 & \\
\hline & & & & Total & 275,1 & & \\
\hline
\end{tabular}

1 - ns e s, respectivamente não significativo e significativo pelo teste de t ao nível de $5 \%$ de probabilidade. 
reduções na produtividade e qualidade dos bulbos. Caso fossem adotados estes níveis para a cv. EMPASC 351 Seleção Crioula, as pulverizações teriam sido feitas entre os níveis de 12 e 30 tripes/ planta. As variações na determinação de nível de dano econômico de tripes em cebola devem-se às diferentes condições de realização de cada trabalho, tais como cultivar, práticas de manejo de lavoura, características edafoclimáticas, além da interação inseto-planta.

De acordo com os níveis de dano econômico encontrados no presente trabalho, conclui-se que é possível tolerar até 10 ninfas por planta antes e 30 após a formação do bulbo sem prejudicar a produtividade, ou até mesmo suprimir o controle químico. Suman \& Wahi (1981) sugeriram uma escala com diferentes níveis de infestação: 1= leve (número igual ou menor que 5 tripes/planta); $2=$ moderada $(10$ a 15 tripes/planta $)$ e $3=$ severa (número maior ou igual que 20 tripes/planta). De acordo com esta escala, neste trabalho foi possível a ocorrência de infestações moderadas antes e severa após a formação do bulbo, sem perdas significativas na produtividade e até mesmo a não adoção do controle químico. Ao confrontar os resultados obtidos com a realidade prática do agricultor catarinense conclui-se que seria possível reduzir o uso de inseticidas em cebola para o controle do tripes. Entretanto, os níveis de matéria orgânica dos solos na região do Alto Vale são relati- vamente baixos, a rotação de culturas não é praticada e a irrigação é pouco adotada, o que dificulta esse processo. Assim, para reduzir a aplicação de inseticidas em cebola, é preciso adotar práticas adequadas de manejo do solo e irrigação, para que as plantas tolerem o dano causado pelo ataque de tripes.

\section{AGRADECIMENTOS}

Aos técnicos agrícolas José D. Petri, Marcelo Pitz e suas equipes, aos laboratoristas Adilson L. Petry, Adriana M. S. Campos, Cleide Tefen, ao responsável técnico da Estação Agrometeorológica da EPAGRI, Ituporanga, SC, Vinícius C. Mello, pelo fornecimento de dados. Ao FEPA (Fundo de Estímulo à Pesquisa Agropecuária) pelo apoio financeiro.

\section{LITERATURA CITADA}

DOMICIANO, N.L.; OTA, A.Y.; TEDARDI, C.R Momento adequado para controle químico de tripes, Thrips tabaci Lindeman, $1888 \mathrm{em}$ cebola, Allium cepa L. Anais da Sociedade Entomológica do Brasil, Porto Alegre, v. 22, n. 1, p. $77-83,1993$.

EMPASC/ACARESC. Sistema de produção para cebola-Santa Catarina (2 ${ }^{\text {a }}$ revisão) Florianópolis: 1991, 51 p. (Sistemas de Produção 16).

HOFFMANN, M.P.; PETDZOLDT, C.H.; MACNEIL, C.R.; MISHANEC, J.J.; ORFANEDES, M.S.; YOUNG, D.H. Evaluation of an onion thrips pest management program for onions in New York. Agriculture, Ecosystems and Environment, v. 55, n. 1, p. 51 - 60, 1995.
FOURNIER, F.; BOIVIN, G.; STEWART, R.K. Effect of Thrips tabaci (Thysanoptera: Thripidae) on yellow onion yields and economic tresholds for its management. Journal of Economic Entomology, v. 88, n. 5, p. 1401 - 1407, 1995.

GOMES, F.P. Curso de Estatística Experimental. 12 ed. Piracicaba: Nobel, 1987. 476 p.

GONÇALVES, P.A.S.; GUIMARÃES, D.R. Controle do tripes da cebola. Agropecuária Catarinense, Florianópolis, v. 8, n. 2, p. 44 46, 1995.

GONÇALVES, P.A.S. Determinação de danos de Thrips tabaci Lind. em cultivares de cebola. Pesquisa Agropecuária Brasileira, Brasília, v. 31, n. 3, p.173 - 179, março 1996.

LORINI, I.; FERRETO, M. Avaliação de danos de Thrips tabaci Lindeman, 1888 (Thysanoptera, Thripidae) na cultura da cebola. Anais da Sociedade Entomológica do Brasil, Porto Alegre, v. 20, n. 2, p. 271 - 275, 1991.

LORINI, I.; TORRES L.; GUIMARÃES, D.R. Flutuação populacional de tripes na cultura da cebola. Florianópolis: EMPASC, 1986. 4 p. (EMPASC. Pesquisa em Andamento, 62).

SHELTON, A.M.; NYROP, J.P.; NORTH, R.C.; PETZOLDT, C.; FOSTER., R. Development and use of a dynamic sequential sampling, program for onion thrips, Thrips tabaci (Thysanoptera: Thripidae), on onions. Journal of Economic Entomology, v. 80, n. 5, p. 1051 - 1056, 1987.

Suman, C.L.; Wahi, S. Sequential sampling plan for the onion thrips, (Thrips tabaci L.). Entomon., v. 6, n. 3, p. 265 - 269, 1981.

YOKOYAMA, S.; THOMAZELLI, L.F.; GUIMARÃES, D.R.; MULLER, J.J.V.; SILVA, A.C.F. da; VIZZOTTO, V.J.; ZANINI NETO, J.A.; BECKER, W.F.; BIASI, J. Nova cultivar de cebola EMPASC 351-Seleção Crioula. Florianópolis, EMPASC, 1984. 14 p. (EMPASC. Comunicado Técnico, 69). 